\title{
Corrosion Failure Cause Analysis of Buried Pipelines in Oil and Gas Stations
}

\author{
Bihuang Su ${ }^{1,2, *}$, Yanjun Zhang ${ }^{1,2}$, Guibai Huang ${ }^{1,2}$, Zhitao Wang ${ }^{1,2}$, Ran Liu ${ }^{1,2}$ \\ ${ }^{1}$ CNPC Engineering Technology Research Company Limited, Tianjin 300451, China; \\ ${ }^{2}$ Key Laboratory of Tubular Goods Engineering, CNPC-Research Division of Anti-Corrosion Coating and Thermal Insulation Structure, \\ Tianjin 300451, China
}

\begin{abstract}
Objective: To explore the failure cause of buried pipelines in an oil and gas station. Method: The chemical elements and metallographic structure of the failed pipes were analyzed to evaluate whether the pipe body meets the requirements of relevant standards; the morphology and composition of the corrosion products were analyzed to discover the cause of corrosion. Result: The metal surface was rough and full of pitting pits with severe localized corrosion, and no crack of the metallic matrix was found. The corrosion products mainly contain $\mathrm{Fe}_{3} \mathrm{O}_{4}$ and a small amount of $\mathrm{FeCO}_{3}$, wherein $\mathrm{Fe}_{3} \mathrm{O}_{4}$ is the secondary corrosion product formed in the air, and $\mathrm{FeCO}_{3}$ is the corrosion product of $\mathrm{CO}_{2}$ in an oil and gas environment. The surface of the corroded product is loose in structure and easy to peel off, leading to further corrosion of the metallic matrix. Conclusion: The pipeline corrosion failure was caused by $\mathrm{CO}_{2}$-induced corrosion failure in the medium.
\end{abstract}

\section{Introduction}

Oil and gas pipeline corrosion is concerned with the safe operation of oil and gas production. The corrosion is characterized by multi-phase flow corrosion mediums where the oil, gas and water coexist; $\mathrm{CO}_{2}, \mathrm{H}_{2} \mathrm{~S}$ and acidic water are the main corrosion media. $\mathrm{CO}_{2}, \mathrm{H}_{2} \mathrm{~S}$ and high $\mathrm{Cl}$ - are more likely to lead to localized corrosion, and along with higher temperatures of some pipelines, the localized corrosion rate is accelerated, resulting in corrosion failure and bringing hidden hazards to the safe operation of pipelines. It is necessary to carry out sampling

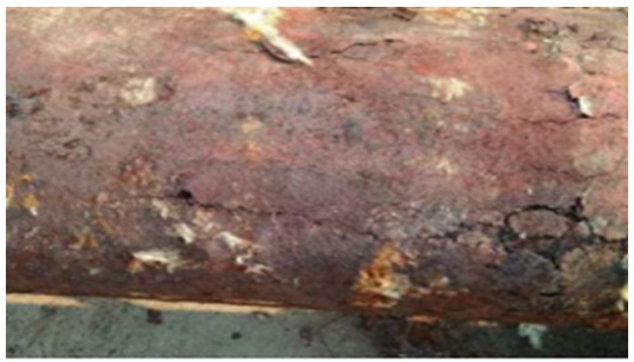

Figure1.External appearance of pipeline

\subsection{Working Condition and Medium}

The design pressure of the pipeline is $4.0 \mathrm{Mpa}$, the operating pressure is $2.20-2.30 \mathrm{MPa}$, the medium is crude oil, the sulfur content is $0.95 \%$, and hydrogen sulfide content is $0.18 \mathrm{mg} / \mathrm{Kg}$. analysis on the failed pipelines, explore the cause of corrosion failure, and take control measures to ensure their safe operation.

\section{Test}

\subsection{Sampling}

The pipelines at the oil and gas station are the $20 \#$ carbon steel pipeline of $\varphi 219 \times 7$. They are located at a $4 \mathrm{~m}$ buried pipeline at the intersection of the production header and the outer oil pipeline.

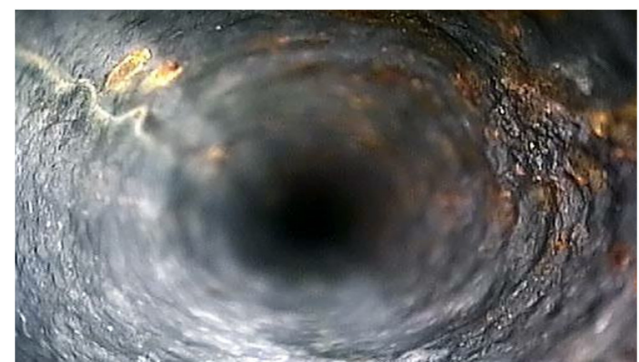

Figure2.Internal appearance of pipeline

\subsection{Test Content}

(1) Material Inspection

Material inspection mainly includes component analysis, metallographic analysis, etc. used to check whether the performance of steel materials meets the

*Corresponding author’ s e-mail: subh.cpoe@cnpc.com.cn 
requirements.

(2) Analysis of Failure Mechanism

It mainly includes the macro morphological observation and corrosion product analysis used to analyze the cause of corrosion failure.

\section{Result and Analysis}

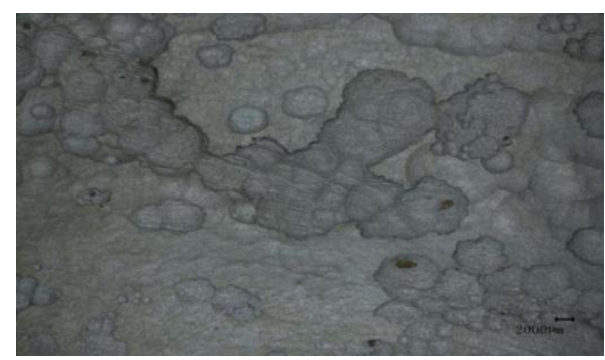

Figure 3. 2D (5x) microscope image of local sample after rust remova

\subsection{Matrix Analysis of 20\# Carbon Steel Pipeline}

Component analysis: Component analysis was carried out

\subsection{Macro Inspection}

By analyzing the pipelines' macro morphology, we found obvious localized corrosion on the inner wall of the pipelines, and the most serious corrosion occurs in the liquid deposition area at the bottom of the pipelines, mainly localized platform-shaped pitting corrosion. There are many corrosion pits with deep depths of up to $4.8 \mathrm{~mm}$. Densely distributed along the axial direction, the pits are mostly elliptical and horizontal.

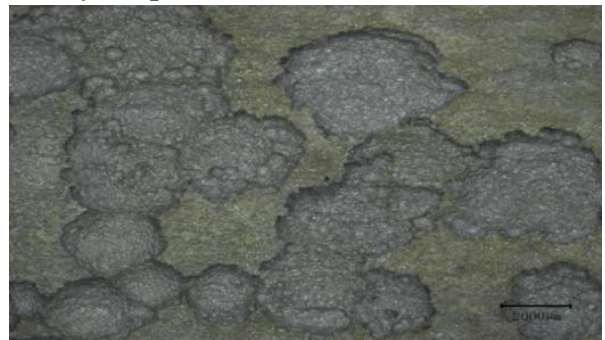

Figure 4. 2D (5x) microscope image of local sample after rust remova

on the test sample, and the spectrum analyzer was used in the test for analysis. It can be seen from the analysis results shown in Table 1 that all element contents in the sample meet the requirements of GB/T 9711-2011. Hence the sample has qualified chemical composition.

Table 1. Element analysis results of 20 \# carbon steel pipe ( $\mathrm{wt} \%$ )

\begin{tabular}{cccccccccccc}
\hline $\mathrm{C}$ & $\mathrm{Si}$ & $\mathrm{Mn}$ & $\mathrm{P}$ & $\mathrm{S}$ & $\mathrm{V}$ & $\mathrm{Nb}$ & $\mathrm{Ti}$ & $\mathrm{Cu}$ & $\mathrm{Ni}$ & $\mathrm{Cr}$ & $\mathrm{Mo}$ \\
\hline$\leq 0.24$ & $\leq 0.45$ & $\leq 1.40$ & $\leq 0.025$ & $\leq 0.015$ & $\leq 0.10$ & $\leq 0.05$ & $\leq 0.04$ & $\leq 0.50$ & $\leq 0.30$ & $\leq 0.30$ & $\leq 0.15$ \\
0.15 & 0.29 & 1.21 & 0.0079 & 0.0047 & 0.002 & 0.037 & 0.025 & 0.089 & 0.021 & 0.065 & 0.011 \\
0.15 & 0.32 & 1.21 & 0.0079 & 0.0047 & 0.002 & 0.036 & 0.023 & 0.089 & 0.021 & 0.066 & 0.011 \\
\hline
\end{tabular}

A high-rate coaxial microscope was used to conduct metallographic observation on the sample. The

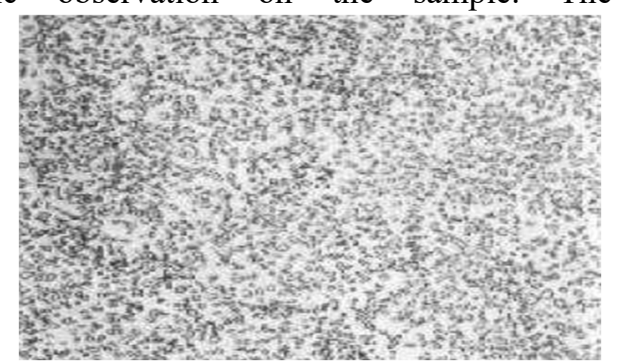

Figure 5. Phase diagram

The pipelines have a metallographic microstructure of massive polygonal ferrite and granular pearlite, with uniform crystalline grains and a small amount distributed in bands, similar to the structure obtained by normalization.

\subsection{Corrosion Product Analysis}

metallographic diagram of the pipe section observed is as follows: ]

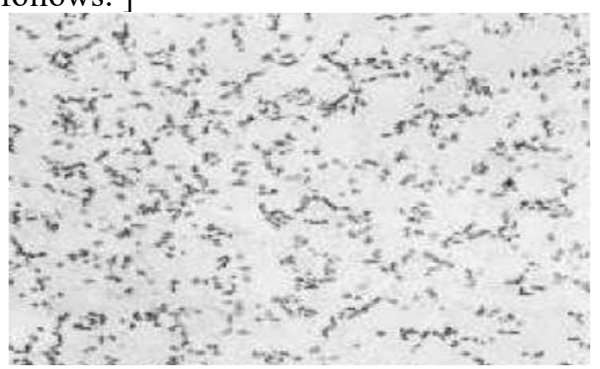

\section{0\# carbon steel (a100Xb500X)}

The measured results of the corrosion products on the inner wall are shown in Figure 6. As can be seen from the XRD analysis results, the materials on the inner wall of the pipeline mainly consist of $\mathrm{Fe}_{3} \mathrm{O}_{4}$ and a small amount of $\mathrm{FeCO}_{3}$, wherein $\mathrm{Fe}_{3} \mathrm{O}_{4}$ is the secondary corrosion product formed in the air, and $\mathrm{FeCO}_{3}$ is the corrosion product of $\mathrm{CO}_{2}$ in the oil and gas environment. 


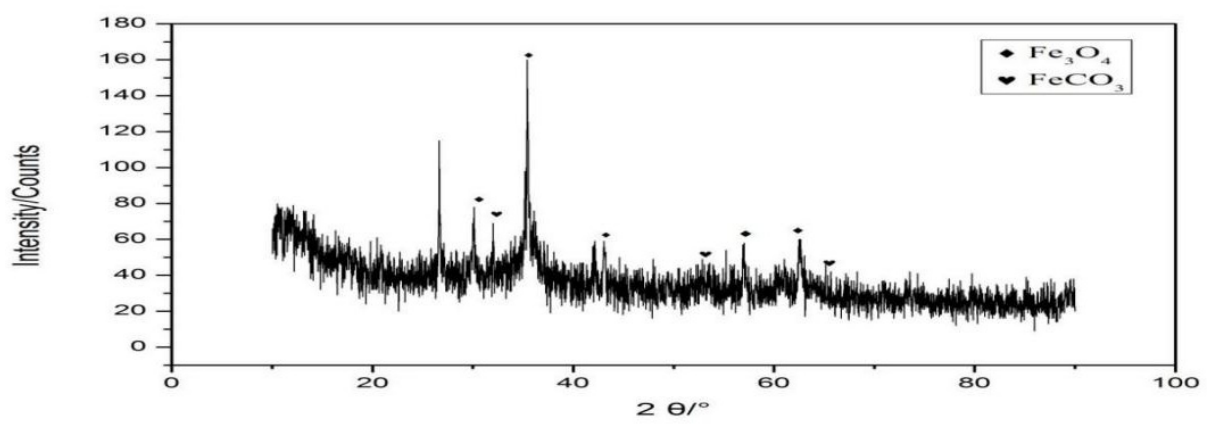

Figure 6. Corrosion products of pipeline inner wall

\subsection{Analysis of Micro Morphology on the Corroded Surface}

The micromorphology and energy spectrum analysis were carried out on the corrosion products The

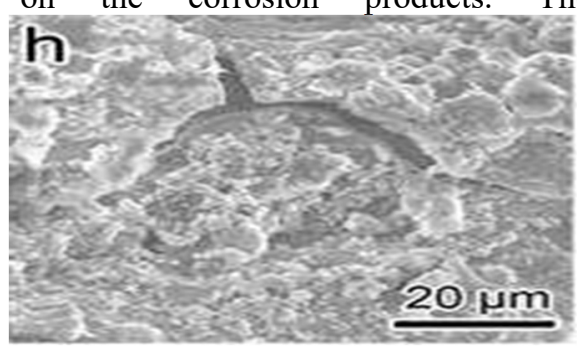

Figure 7. SEM image of samples without derusting micromorphology is shown in Figures 7 and Figures 8, and the energy spectrum is shown in Figures 9 and 10. The component analysis of corrosion products is shown in Tables 2 and 3 . As can be seen from the table, the corrosion products mainly contain $\mathrm{Fe}, \mathrm{O}$ and a small amount of other elements such as calcium chloride.

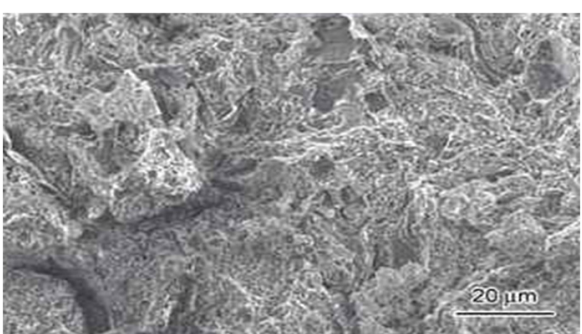

Figure 8. SEM image of samples derusting

Table 2. Content of components in corrosion products of outer wall

\begin{tabular}{|c|c|c|c|c|c|}
\hline element & $\mathrm{C}$ & $\mathrm{O}$ & $\mathrm{Cl}$ & $\mathrm{Mn}$ & $\mathrm{Fe}$ \\
\hline Wt.\% & 2.59 & 11.03 & 1.18 & 1.21 & 83.93 \\
\hline
\end{tabular}

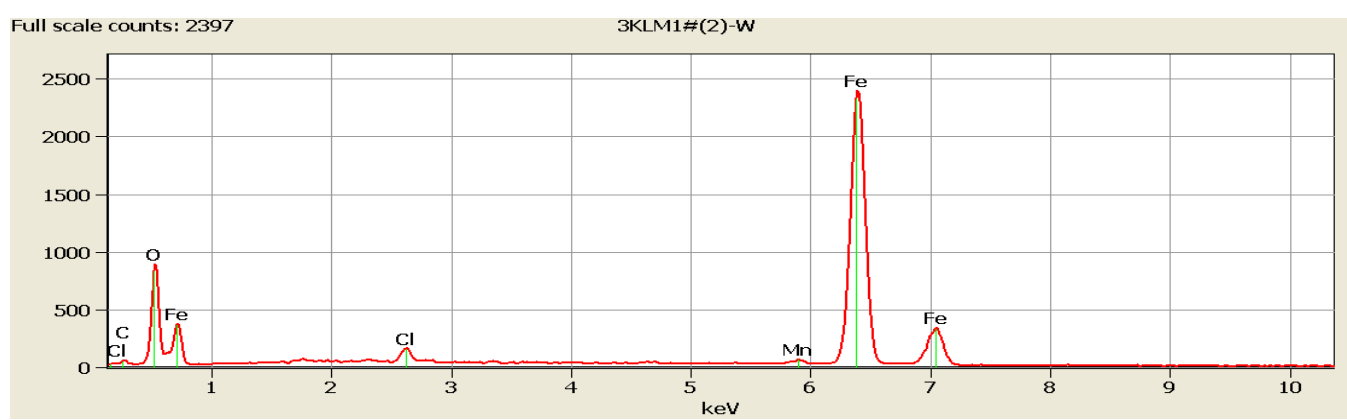

Figure 9. Energy spectrum of corrosion pit in outer wall of pipeline section in zone 2 of heavy oil productio

Table 3. Content of components in corrosion products of inner wall

\begin{tabular}{|c|c|c|c|c|c|c|c|}
\hline element & $\mathrm{C}$ & $\mathrm{O}$ & $\mathrm{Al}$ & $\mathrm{Si}$ & $\mathrm{S}$ & $\mathrm{Mn}$ & $\mathrm{Fe}$ \\
\hline Wt.\% & 8.91 & 16.93 & 0.51 & 1.84 & 2.29 & 0.64 & 67.89 \\
\hline
\end{tabular}

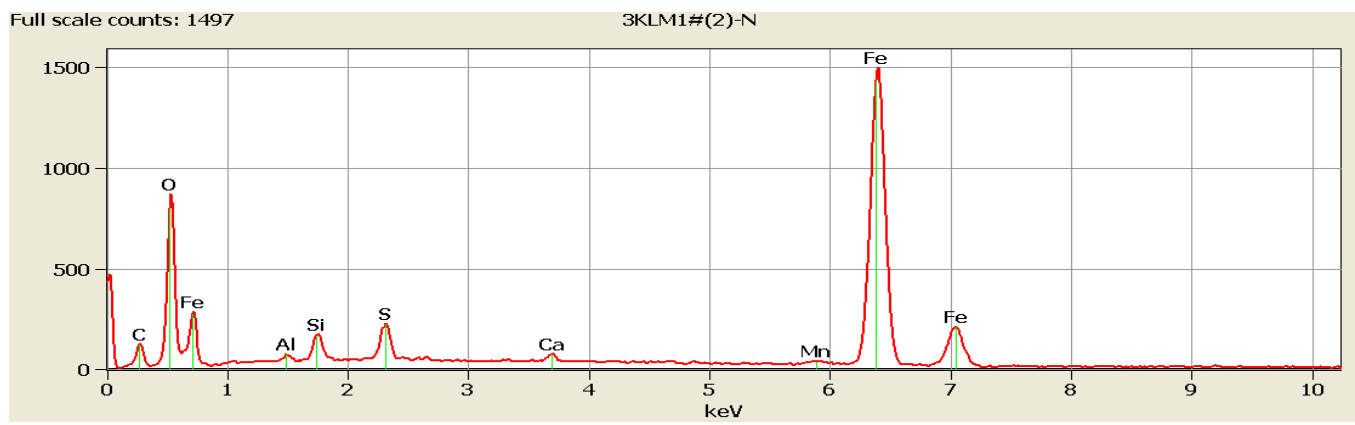

Figure 10. Energy spectrum of corrosion pit in inner wall of pipeline section in zone 2 of heavy oil production 
It can be seen from Figure 7 that the corrosion products are loose in structure with obvious cracks on the surface surrounded by corrosion pits. As shown in Figure 8, the metal surface is rough and full of corrosion pits with severe localized corrosion, and no cracks are found on the metallic matrix. The corrosion products formed are loose in structure, and it is difficult for carbon steel to generate $\mathrm{FeCO}_{3}$ protective film in the medium and low-temperature area $\left(<60^{\circ} \mathrm{C}\right)$. However, a loose product film was formed, which is easy to peel off and cause the matrix to further corrode.

\subsection{Analysis Result}

(1) By cutting and derusting the pipelines, and observing the macro and local morphology of each pipeline's inner wall, we found that the inner wall is severely corroded. The liquid deposit area at the bottom of the pipeline was severed corroded, mainly composed of localized platformshaped pitting corrosion, while other areas are dominated by uniform corrosion, with deeper depths of corrosion pits in severe corrosion area, up to $4.8 \mathrm{~mm}$.

(2) Component analysis showed all element contents in the pipeline meets the standards. According to the metallographic analysis, the metallographic structure mainly consists of ferrite and pearlite, with uniform crystalline grains and a small amount distributed in bands, similar to the structure obtained by normalization.

(3) In terms of corrosion products, the corrosion products of pipeline mainly contain $\mathrm{Fe}_{3} \mathrm{O}_{4}$ and a small amount of $\mathrm{FeCO}_{3}$, wherein $\mathrm{Fe}_{3} \mathrm{O}_{4}$ is the secondary corrosion product formed in the air, and $\mathrm{FeCO}_{3}$ is the corrosion product of $\mathrm{CO}_{2}$ in an oil and gas environment.

(4) As shown in the field emission scanning SEM image, the corrosion products are loose in structure and full of corrosion pits with severe localized corrosion, and no cracks on the metallic matrix. The corrosion products formed are loose in structure, and it is difficult for the carbon steel to generate protective film on the surface in the medium and low-temperature area $\left(<60^{\circ} \mathrm{C}\right)$. However, a loose product film was formed, which is easy to peel off and cause the matrix to further corrode.

\section{Conclusion}

(1) The surface of the corroded pipeline is rough and full of corrosion pits with severe localized corrosion, and no cracks were found on the metallic matrix.

(2) The test results of the elements and metallographic structure of the steel pipe meet the requirements of the standard.

(3) The pipeline corrosion was caused by $\mathrm{CO}_{2}-$ induced corrosion failure in the medium.

\section{References}

1. Li,L.J., Han,W.L.,Yang,Y.H.(2017) Effect of inhibitor type and moisture content on corrosion behavior of carbon steel in $\mathrm{H} 2 \mathrm{~S} / \mathrm{CO} 2$ environment [J]. Corrosion and protection, 38 (10) :795-799
2. Zhong,B. , Chen,Y.Q.,Meng,F.L.(2018) Failure analysis of N80 tubing perforation [J], Corrosion and protection, 39(8): 647-653

3. Zhang,C.Z.(1988) Corrosion and protection of metals [M],Metallurgical Industry Press , beijing, 1988

4. Li,H.L.,Zhao,X.W.(2005) Failure Analysis and integrity management of oil and gas pipeline [J], Physical and chemical examination-physical, 41 (21) : 24-31

5. Li,P.Q.(2005) Failure accident of oil and gas transmission pipeline and typical cases $[\mathrm{J}]$, Welded pipe, 28 (4) : 76-81 